\title{
Induced electromagnetic field on underground metal pipelines running parallel to nearby high voltage $\mathrm{AC}$ power lines
}

\author{
Giovanni Dushimimana ${ }^{*}$, Patrobers Simiyu, Vedaste Ndayishimiye, Emile Niringiyimana and Sefu Bikorimana \\ School of Electrical and Electronic Engineering, North China Electric Power University, Beijing 102206, CHINA
}

\begin{abstract}
High voltage AC (HVAC) power lines can induce significant amount of voltages on underground gas/oil metal pipelines in areas where they share similar Right of Way (RoW), the situation becoming serious particularly in case of fault conditions. Electromagnetic field generated by the HVAC power lines on these pipelines, generate unwanted voltages which present threats to the pipeline and its associated protective equipment such as cathodic systems. The aim of this research is to compute induced voltage on pipeline running parallel to HVAC power lines. The study was conducted using CDEGS software package and revealed that under steady state condition, results were in agreement with GB 68301986 standard. However, high currents are generated under single phase to ground short circuit rising the potential of adjacent soil including the underground pipeline beyond limits. Therefore, the gradient control wire mitigation technique is proposed to reduce these voltages to safe limits.
\end{abstract}

\section{Introduction}

The annual increase in population and growth of industries increase energy demand to record levels. As a result of these, electric power generation companies make efforts to increase their supply to meet the load demand [1]. This goes with the rapid construction of power transmission lines and oil/gas metal pipelines leading to closeness of high voltage transmission lines and metallic pipelines [2] [3]. Consequently, the collocation of pipelines sharing Right of Way (RoW) with high voltage power line arise where the pipelines may be subject to Electromagnetic Interference (EMI) from High voltage AC (HVAC) power lines [4] [5]. The EMI also affects the safety of personnel who may be in direct contact with the exposed part of the pipeline [6]. Thus, it is not surprising to be concerned with the study of AC interference aimed at understanding their impacts and applicable mitigation measures.

Generally, interference between power transmission lines and pipelines comprise an inductive, a capacitive and a conductive part. The inductive component results from the magnetic field produced by the power line, during steady state and fault operating conditions or in situation of unbalanced phase currents [7] [8]. Capacitive coupling only affects the pipeline installed above the ground due to the capacitance formed between the AC transmission lines and the pipeline. Conductive interference occurs when lightning strikes a transmission line, or when there is a phase to ground fault. Once this happens, the tower base potential and that of the neighbouring soil with regard to the remote earth rises which can result in arcing of the pipeline coating [9] [2].
Over the past years, researchers examined the AC interference problem resulting into various reports, papers, and standards. Initial attempts to study the interference problem started with the Carson's relations in 1926. The Carson's relations provided a solution to very low frequencies and/or perfectly conducting earth [5]. However, the Carson's formula was further modified by Wise in 1934 and became applicable for high frequency. In 2015, Ametani et al, further investigated how Carson's formulae deal with displacement currents based on a stratified earth impedance by adopting an earth-return admittance and showed that Carson's formulae can deal with displacement currents when the relative earth permittivity is one [10].

This research investigates different parameters that influence voltage induced on underground metal pipeline running parallel to neighbouring high voltage $\mathrm{AC}$ power lines hence affecting the pipeline integrity. The study will be conducted using a specialized user-friendly software called Current Distribution, Electromagnetic Fields, Grounding and Soil Structure Analysis (CDEGS) developed by SES to compute voltage induced on the pipeline under steady state and single phase to ground fault condition. Furthermore, the mitigation system for EMI will be applied.

\section{Description of the studied case}

A $500 \mathrm{kV} / 50 \mathrm{~Hz}$ double vertical circuit power line runs parallel to an underground metal pipeline for $20 \mathrm{Km}$ and extends $5 \mathrm{~km}$ beyond the parallel routing at both ends. The phase conductors are arranged in 4-bundle conductors per phase, with bundle spacing of $400 \mathrm{~mm}$. The overhead earth conductor has an equivalent radius of

* Corresponding author: dushimimana36@gmail.com 
$5.7 \mathrm{~mm}$. The underground metal pipeline is buried at 1.6 $\mathrm{m}$ below the ground, with inner radius of $485 \mathrm{~mm}$ and outer radius of $505 \mathrm{~mm}$. The thickness of the anticorrosion layer is $3 \mathrm{~mm}$ while the resistivity of the anticorrosion layer equals to $3 * 10^{4} \Omega \mathrm{m}^{2}$. The soil is assumed to be homogenous with a resistivity of $100 \Omega \mathrm{m}$ whereas the horizontal distance between the power line and the pipeline is $200 \mathrm{~m}$. Phase conductor location on the power line towers regarding the underground pipeline are as described in Fig. 1.

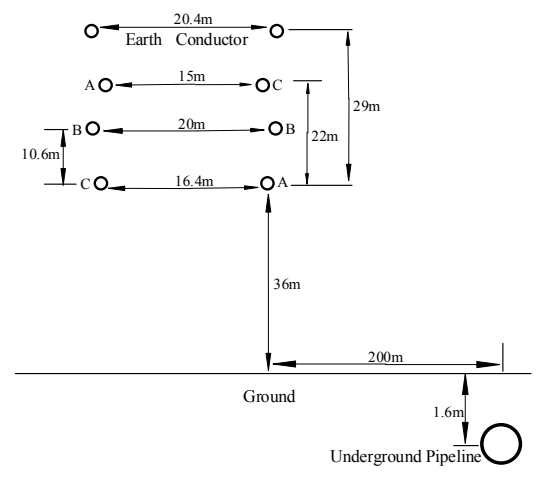

Fig. 1. Cross section of the power line and underground pipeline.

\section{Analysis of induced electromagnetic field}

\subsection{EMI evaluation method}

Generally, conventional circuit method (CCM) and electromagnetic field method (EFM) are the two techniques commonly used to evaluate EMI generated by power lines running parallel to pipelines [11] [12]. The application of the CCM involves the calculation of inductive and conductive interference separately. To model a circuit, many line parameters need to be calculated and to get the overall interference, the inductive and conductive components have to be added together which is a time-consuming process. However, this method provides accurate results for well coated pipelines but the accuracy of the results reduce in case of poor pipeline coating or when gradient control wires are mounted along the pipeline [13] [14].

In case of EFM evaluation, a model of conductor system comprising the underground pipeline, the transmission line phase wires, and the overhead earth conductors together with the towers and grounding systems, is designed. EFM computes the overall interference in one step, preventing the computation of the inductive and conductive components separately as it is done in CCM [15]. A powerful modelling software package called CDEGS released by SES-tech has an integrated HIFREQ module that uses EFM. This has been extensively useful in the calculation of AC interferences between high voltage power lines and neighbouring metal constructions [16] [11]. It is in this case that this software package is used in this paper to model and analyse these electromagnetic interferences.

\subsection{Steady-State operating condition}

In steady-state operating condition, induced voltage on the pipeline is generally affected by power line and pipeline parameters, configuration and soil resistivity surrounding the pipeline. In this section different parameters affecting the pipeline integrity are analysed during normal operating condition with a balanced load current of $2 \mathrm{kA}$.

Fig. 2 shows the effect of balanced load current on the induced voltage. It can be observed that induced voltage increases with the increase in the load current. This is caused by a high magnetic field created around the power line conductors when the load current is increased leading to a high induced voltage on the pipeline. It is also found that, the maximum voltage appears at the extremities of parallelism, and this voltage decreases exponentially reaching zero at the center of the pipeline which is in agreement with the author in [17].

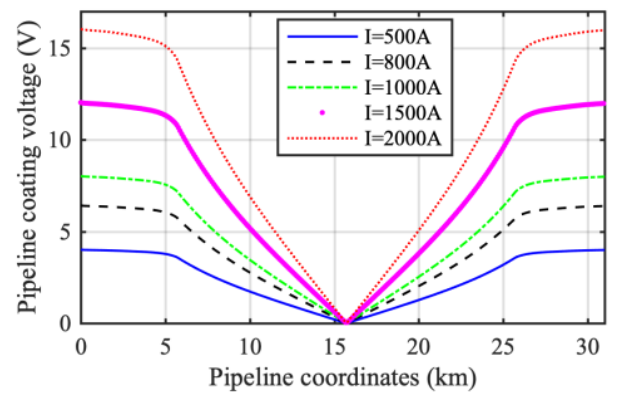

Fig. 2. Effect of load current.

Fig. 3 shows the influence of parallel length when varying between 5 and $30 \mathrm{Km}$. The analysis shows that the maximum induced voltage on the pipeline increases progressively with the increase of the pipeline length. It can be observed that when the maximum voltage reaches a certain value, it starts to decrease lightly towards a stable value. At the point where the highest voltage occurs is equivalent to the saturated length of the pipeline. This is the pipeline length that makes the maximum induced voltage on the pipeline reach its maximum value.

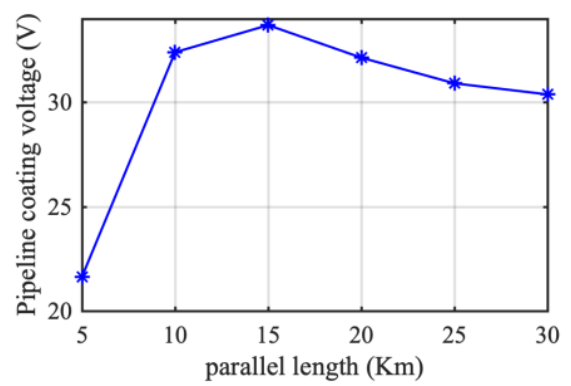

Fig. 3. Effect of parallel length.

Fig. 4 shows the variation of the induced voltage on the pipeline under different phase sequence arrangement modes of the double vertical circuit power line. During the simulation, the left side is arranged in $\mathrm{ABC}$ sequence while the right-side is configured in 6 different phase 
sequences ( $\mathrm{ABC}, \mathrm{ACB}, \mathrm{BAC}, \mathrm{BCA}, \mathrm{CAB}, \mathrm{CBA})$. It can be seen that the configuration of the power line in different phase sequence arrangements has great influence on the voltage induced on the pipeline. ABC$\mathrm{BCA}$ phase sequence induces lower voltage on the pipeline than other configurations.

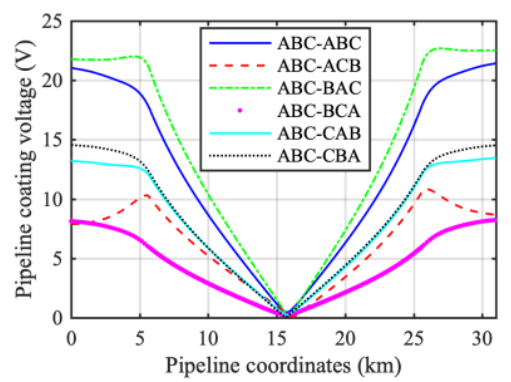

Fig. 4. Effect of phase sequence.

Fig. 5 shows the maximum induced voltage on the pipeline with different soil resistivities and cross-over angles. The earth resistivities for the study include 100 $\Omega \mathrm{m}, 300 \Omega \mathrm{m}, 500 \Omega \mathrm{m}, 800 \Omega \mathrm{m}, 1000 \Omega \mathrm{m}, 1200 \Omega \mathrm{m}$ and $2000 \Omega \mathrm{m}$ respectively. The pipeline crosses the transmission line at different angles: $15^{0}, 25^{0}, 30^{\circ}, 45^{\circ}$, $60^{\circ}, 75^{\circ}$, and $85^{\circ}$ and extends far away at both ends. It can be observed that the angle at which the pipeline crosses the power line has a great influence on the voltage induced on the pipeline. The maximum induced voltage is inversely proportional to the cross-over angle; the smaller the angle the higher the induced voltage. Additionally, it can be observed that the induced voltage expands with increase in the earth resistivity.

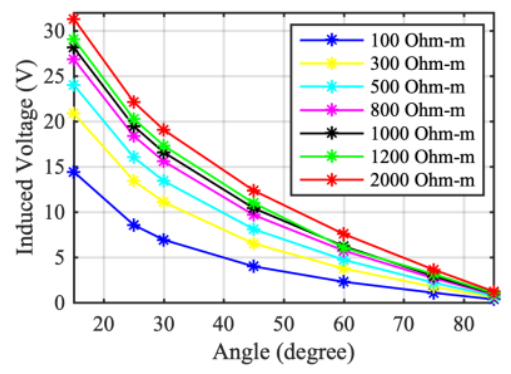

Fig. 5. Effect of cross-over angle.

\subsection{Fault condition}

Generally, the occurrence of faults in the system increase the effect of $\mathrm{AC}$ interference induced in the nearby metallic structures. When a single-phase to ground shortcircuit fault arises in the power line, the fault currents travel down to the ground via the tower grounding structure causing the neighbouring ground potential to increase. In this case, the transmission line routes parallel to the pipeline for a length of $10 \mathrm{Km}$, and then extends beyond the parallel routing at both ends for a length of $2.5 \mathrm{Km}$. The lateral distance between power line and the pipeline is $400 \mathrm{~m}$ while other parameters remain the same as in the steady state condition.
Fig. 6 shows voltage induced on the pipeline when a single phase to ground fault occurs on each phase of the transmission line. It can be observed that the level of fault current differs according to the faulted phase. In the model, phase conductors are arranged in $\mathrm{ABC}$ sequence from up to down. It is observed that the highest voltage is induced on the lower conductor and decreases as we move up. From the results, we can deduce that the closer the phase conductor is to the ground, the higher the induced voltage on the pipeline extremities. This is caused by the distance travelled by the fault currents since these currents vary with tower grounding resistance. The longer the distance, the higher the resistance thus, low voltage induced on the pipeline and vice versa. During the fault condition, the classical "V" shaped curve is obtained because the influence of the electromagnetic field produced by the unfaulted phases is very small compared to the one produced by the faulted phase [18].

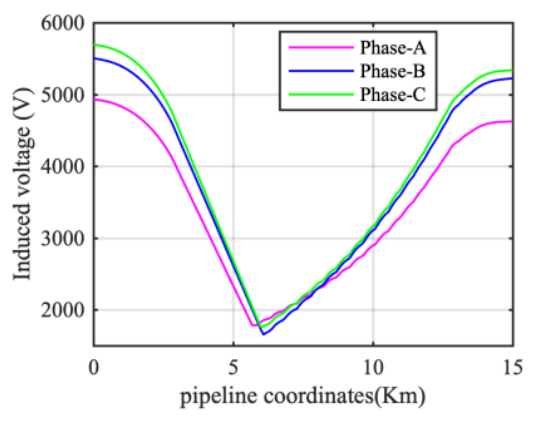

Fig. 6. Effect of short circuit fault on different phases.

From Fig. 7, it can be observed that when the lateral distance D increases from $100 \mathrm{~m}$ to $600 \mathrm{~m}$, the voltage induced on the pipeline decreases by $62 \%$. The magnetic field generated by the transmission lines during fault condition is directly proportional to the fault current flowing in the faulted phase conductor to the ground and inversely proportional to the lateral distance separating the power transmission line and the pipeline. Apparently, the larger the separation distance between the power transmission line and the neighbouring pipeline, the lower the voltage induced on the pipeline.

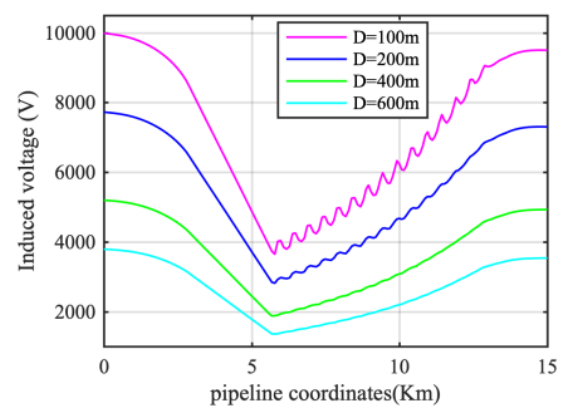

Fig. 7. Effect of distance between power line and pipeline.

Fig. 8 shows the maximum induced voltage on the pipeline as a function of earth resistivity. During the study, the following earth resistivity values $100 \Omega \mathrm{m}, 300$ $\Omega \mathrm{m}, 500 \Omega \mathrm{m}, 1000 \Omega \mathrm{m}$, and $2000 \Omega \mathrm{m}$, respectively were 
considered. It can be observed that, the maximum induced voltage on the pipeline increases gradually with the increase in the earth resistivity. It can also be noted that when the earth resistivity is less than $1000 \Omega \mathrm{m}$, the maximum voltage changes significantly with the increase in the earth resistivity. However, when the earth resistivity is higher than $1000 \Omega \mathrm{m}$, the change in the maximum voltage changes slightly.

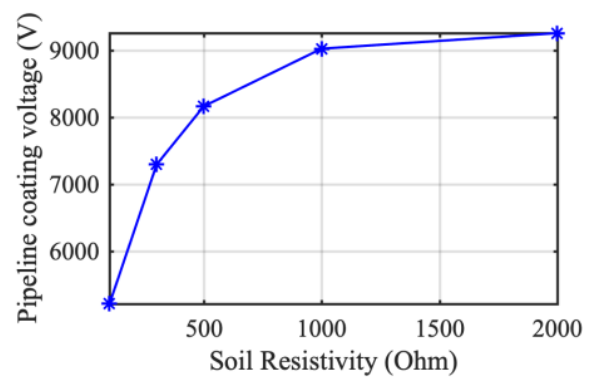

Fig. 8. Effect of earth resistivity.

During the analysis, the values of the grounding resistance were $1 \Omega \mathrm{m}, 5 \Omega \mathrm{m}, 10 \Omega \mathrm{m}, 15 \Omega \mathrm{m}$, and $20 \Omega \mathrm{m}$, respectively. Fig. 9 illustrates the effect of grounding resistance on the maximum value of the induced voltage on the pipeline. It can be observed that this value declines with the increase in the grounding resistance value. When a fault occurs in the power line, the high currents generated flow into the earth through the grounding structures of the tower. The grounding resistance plays a vital role in the analysis of the induced voltage on the pipeline. Certainly, the larger the grounding resistance, the lower the short-circuit current thus, inducing lower voltage on the pipeline.

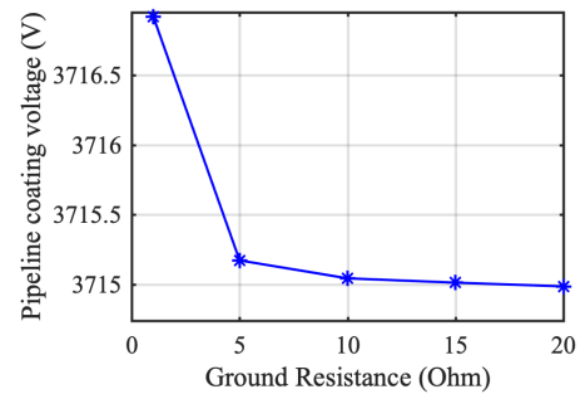

Fig. 9. Effect of tower grounding resistance.

\section{Application of mitigation systems}

Mitigation systems aim to reduce induced voltage on the pipeline to acceptable limits. In this section gradient control wire mitigation system is applied to reduce induced voltage to safe limits as specified in GB 68301986 and ITU-T DL/T 5033-2006 standards. Fig. 10 shows the application of the mitigation system in voltage profiles in Fig. 7. The worst-case scenario involving $\mathrm{D}=100 \mathrm{~m}$ and the least severe case with $\mathrm{D}=600 \mathrm{~m}$ are considered. The system consists of a pair of bare copper wires placed in parallel with the pipeline at a depth of $1.6 \mathrm{~m}$. The copper wires are connected to the pipeline at both ends. It can be observed that the maximum voltage reaches $2621 \mathrm{~V}$ (26.24\% of the value without mitigation system) when the horizontal distance between the pipeline and power line equals $100 \mathrm{~m}$. When the horizontal distance between pipeline and power line equals $600 \mathrm{~m}$, the maximum induced voltage is $818 \mathrm{~V}$ $(8.18 \%$ of the value without mitigation system). In this case, the mitigation reduces the induced voltage significantly at different levels depending on the horizontal distance between the pipeline and the power line. Therefore, this research proposes the pipelines to be located $600 \mathrm{~m}$ from the power line and gradient control wire installed to limit the voltages to safe limits hence minimize the effect of the EMI.

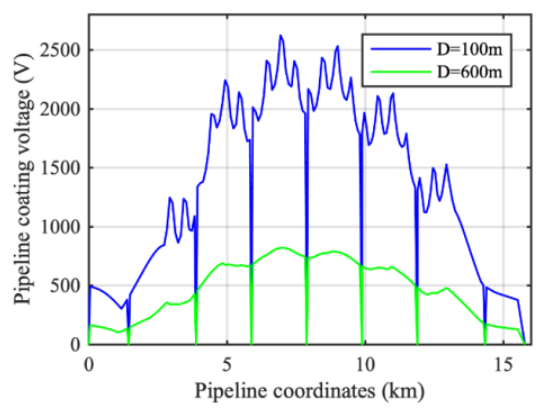

Fig. 10. Induced voltage along the pipeline under fault condition: gradient control wires present.

\section{Conclusion}

This research investigated different parameters that influence voltage induced on underground metal pipeline running parallel to neighbouring high voltage AC power lines under steady state and fault conditions using CDEGS. The findings show that under steady state condition: (1) the induced voltage increases with the increase in the load current. (2) the maximum voltage appears at the saturated length of the pipeline. (3) ABC$\mathrm{BCA}$ phase sequence induces the lowest voltage on the pipeline. (4) the maximum induced voltage is inversely proportional to the cross-over angle; the smaller the angle the higher the induced voltage. In case of single phase to ground fault: (1) the closer the phase conductor is to the ground, the higher the induced voltage on the pipeline extremities. (2) the larger the separation distance between the power transmission line and the pipeline, the lower the induced voltage. (3) the maximum induced voltage on the pipeline increases with the increase in the earth resistivity. (4) the induced voltage declines with the increase in the grounding resistance value. The gradient control wire mitigation system reduces the induced voltage by $26.24 \%$ and $8.18 \%$ respectively when applied to the worst-case scenario involving $\mathrm{D}=100 \mathrm{~m}$ and the least severe case with $\mathrm{D}=600 \mathrm{~m}$. The EMI has significant effects on the underground pipeline, severe effects such as higher the induced voltage on the pipeline extremities must be mitigated. Therefore, this research proposes the pipelines to be located $600 \mathrm{~m}$ from the power line and gradient control wire installed to limit the voltages to safe 
limits as specified in GB 6830-1986 and ITU-T DL/T 5033-2006 standards.

\section{References}

1. K. B. Adedeji, A. A. Ponnle, B. T. Abe, and A. A. Jimoh, "Effect of increasing energy demand on the corrosion rate of buried pipelines in the vicinity of high voltage overhead transmission lines, " in Intl Aegean Conference on Electrical Machines \& Power Electronics, (2015), pp. 299-303.

2. L. Qi, H. Yuan, L. Li, and X. Cui, "Calculation of Interference Voltage on the Nearby Underground Metal Pipeline due to the Grounding Fault on Overhead Transmission Lines," IEEE Trans. Electromagn. Compat., vol. 55, no. 5, pp. 965-974, (2013).

3. M. M. Saied, "The Capacitive Coupling Between EHV Lines and Nearby Pipelines," IEEE Trans. power deliv., vol. 19, no. 3, pp. 1225-1231, (2004).

4. B. K. Shane Finneran, "Criteria for Pipelines CoExisting with Electric Power Lines," INGAA Foundation Inc, Dublin, (2015).

5. G. C. Christoforidis, D. P. Labridis, and P. S. Dokopoulos, "Inductive Interference on Pipelines Buried in Multilayer Soil Due to Magnetic Fields From Nearby Faulted Power Lines," IEEE Trans. Electromagn. Compat., vol. 47, no. 2, pp. 254-262, (2005).

6. M. Chen, S. Liu, J. Zhu, C. Xie, H. Tian, and J. Li, "Effects and Characteristics of AC Interference on Parallel Underground Pipelines Caused by an AC Electrified Railway," Energies, vol. 11, no. 9, pp. 124, (2018).

7. G. C. Christoforidis, D. P. Labridis, and P. S. Dokopoulos, "A Hybrid Method for Calculating the Inductive Interference Caused by Faulted Power Lines to Nearby Buried Pipelines," IEEE Trans. power deliv., vol. 20, no. 2, pp. 1465-1473, (2005).

8. M. A. H. E.-G. Ossama E. Gouda, Adel Z. El Dein, "Effect of electromagnetic field of overhead transmission lines on the metallic gas pipe-lines," Electr. Power Syst. Res., vol. 103, pp. 129-136, (2013).

9. R. Djekidel and D. Mahi, "Capacitive Interferences Modeling and Optimization between HV Power
Lines and Aerial Pipelines,” Int. J. Electr. Comput. Eng., vol. 4, no. 4, pp. 486-497, (2014).

10. A. Ametani et al., "A Study on High-Frequency Wave Propagation along Overhead Conductors by Earth-Return Admittance/Impedance and Numerical Electromagnetic Analysis," in International Conference on Power Systems Transients, (2015).

11. L. A. Denisa Stet, Dan D. Micu, Levente Czumbil, "Effects of Power Line Operating Conditions on Nearby Gas Pipelines," in World Energy System Conference, (2012), no. 3, pp. 731-736.

12. D. D. Micu, L. Czumbil, L. Darabant, and A. Ceclan, "Simulation of interferences between power lines and gas pipelines in unbalanced phase currents state," COMPEL Int. J. Comput. Math. Electr. Electron. Eng., vol. 31, no. 4, pp. 1178-1189, (2008).

13. H. M. A.-R. A. H. Al-Badi, "Simulation of Electromagnetic Coupling on Pipelines close to Overhead Transmission Lines: A Parametric Study," J. Commun. Softw. Syst., vol. 1, no. 2, pp. 116-125, (2005).

14. J. Ma and F. P. Dawalibi, "Recent Advances in Electromagnetic Interference Analysis in Common Corridors," in 4th Asia-Pacific Conference on Environmental Electromagnetics, (2006), no. 2, pp. 409-418.

15. D. Stet, L. Czumbil, D. D. Micu, and O. Miron, "Corossion Evaluation and Mitigation on Metallic Pipelines," in International Conference and Exposition on Electrical and Power Engineering, (2012), pp. 554-559.

16. J. Ma, Z. Wu, M. Hu, X. Dou, Y. Wang, and H. Chen, "Research on the Numerical Accuracy of Equipotential Ground Model Based on Method of Moment," WSEAS Trans. Power Syst., vol. 11, pp. 52-57, (2016).

17. D. D. Micu, G. C. Christoforidis, and L. Czumbil, "AC interference on pipelines due to double circuit power lines: A detailed study," Electr. Power Syst. Res., vol. 103, pp. 1-8, (2013).

18. L. Czumbil, Ş. Denisa, D. D. Micu, and Ț. Vasile, "Stream Gas Pipeline in Proximity of High Voltage Power Lines. Part II-Induced Voltage Evaluation," in 47th International Universities Power Engineering Conference, (2012), pp. 1-6. 